\title{
Stretchable and recoverable acrylate-based pressure sensitive adhesives with high adhesion performance, optical clarity, and metal corrosion resistance
}

\author{
Ju Hak Lee ${ }^{\mathrm{a}, 1}$, Jintae Park ${ }^{\mathrm{a}}$, Min Hoon Myung ${ }^{\mathrm{a}}$, Myung-Jin Baek ${ }^{\mathrm{a}}$, Hak-Sun Kim ${ }^{\mathrm{b}}$, \\ Dong Woog Lee La $^{a}$ \\ ${ }^{a}$ School of Energy \& Chemical Engineering, Ulsan National Institute of Science and Technology (UNIST), Ulsan 44919, Republic of Korea \\ ${ }^{\mathrm{b}}$ Department of Electrical Engineering, Ulsan National Institute of Science and Technology (UNIST), Ulsan 44919, Republic of Korea
}

\section{H I G H L I G H T S}

- Acrylate-based stretchable, optically transparent PSAs are synthesized.

- Synthesized PSAs exhibit high adhesion strength and rapid strain reversibility.

- PSAs show high optical transparency in the visible regime.

- PSAs exhibit excellent compatibility with the corrosion sensitive layer.

- Pre-strain strategy enables instantaneous recovery of PSAs upon strain relaxation.

\section{A R T I C L E IN F O}

\section{Keywords:}

Pressure sensitive adhesive

Reversibility

Peel adhesion

Acrylic acid

Metal corrosion

\section{G R A P H I C A L A B S T R A C T}

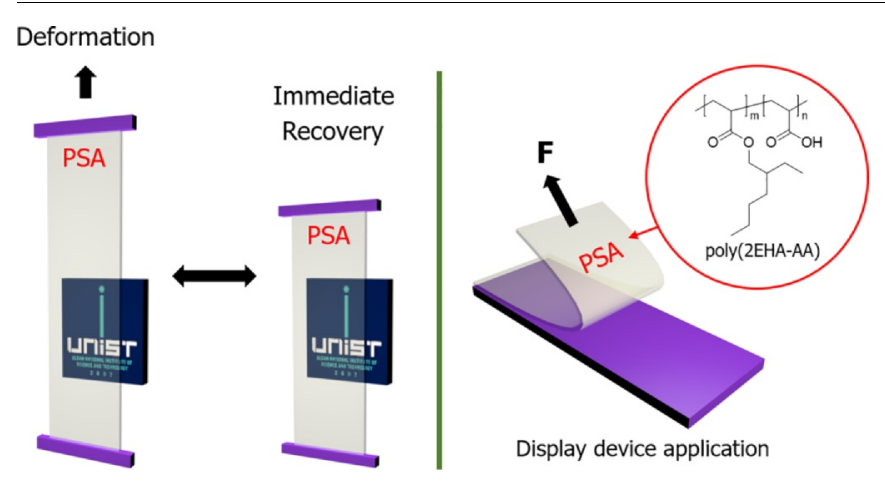

\begin{abstract}
A B S T R A C T
In the era of the Internet of Things, digital displays play a critical role in human-machine interfaces. In particular, displays applied to contemporary devices such as flexible smart watches and foldable/rollable electronics underline the need for pertinent materials and device technologies to fulfill their designed functions. However, despite the technical advancements of electronic components such as stretchable/flexible electrodes and flexible backplanes, their proper assembly remains a challenge. Herein, we report the compositional effect of acrylic acid (AA) on the physical properties of as-synthesized pressure sensitive adhesives (PSAs), especially on their adhesion performance in terms of wettability and peel adhesion. Accordingly, an empirical criterion for intimate wetting is proposed based on the storage modulus of the PSAs. In this study, the PSA with the best adhesion performance was evaluated for its viscoelastic properties and suitability for specific applications. The results demonstrated the strain-dependent conformational recovery of the adhesive; the implementation of the prestrain strategy enables rapid strain reversibility at $25 \%$ of the total strain. Furthermore, the AA-incorporated PSAs exhibited remarkable metal corrosion resistance as well as high optical clarity. Thus, this fundamental study of stretchable PSAs can provide useful guidance for the development of advanced PSAs that can be used in a wide range of applications involving display devices.
\end{abstract}

\footnotetext{
* Corresponding author.

E-mail address: dongwoog.lee@unist.ac.kr (D.W. Lee).

${ }^{1}$ Current address: Standard Energy Co., Ltd., Daejeon 34014, Republic of Korea.
} 


\section{Introduction}

Displays are vital components of standard human-machine interfaces in contemporary electronic devices, as they provide machine data to users and user feedback/response to machines $[1,2]$. Consequently, the use of related materials and display technologies is an important requisite for the development of advanced electronic devices. The structures of displays have been evolving from a stiff and rigid plate shape (e.g., SAMSUNG Galaxy S1 and Apple iPhone 1) to a flexible or curved form (e.g., SAMSUNG Galaxy S9 Plus and Nubia Alpha Smart watches); this has been aimed at increasing the level of intimacy between the user and the device. Although flexible/stretchable displays are desirable in everyday appliances such as televisions, mobile phones, and smart watches for user convenience, they are indispensable for specialized devices such as epidermal electronics comprising designs that can be utilized in biomedical applications and healthcare systems $[1,3,4]$.

In accordance with the advancements made in display technologies, pressure sensitive adhesives (PSAs) have been widely used for assembling display components $[5,6]$; this is due to their flexibility, high viscoelasticity [7], and ability to minimize the discrepancies in refractive index by filling air gaps, thereby contributing to the clarity of the image displays [8]. Although PSAs form adhesive bonds with their substrates upon the application of a low pressure, they can be peeled off without the formation of any residue in most cases [9]. Moreover, as they do not require post-treatments such as solvent evaporation and curing using heat or chemicals, PSAs are considered advantageous over all other classes of adhesives [10]. Among these different classes, acrylate-based PSAs are widely used in various fields as they facilitate the formation of diverse material designs; this is because they are composed of a variety of monomers with different functional groups [11,12]. However, the intrinsic properties of conventional PSAs hinder their applicability as stretchable adhesives, which is a result of their rigidity, irreversibility upon stretching, or optical opaqueness.

Therefore, the physical properties of acrylic PSAs have been intensively investigated for their utilization in stretchable and flexible displays $[5,7,13]$; the results revealed a tradeoff relationship between peel adhesion and strain reversibility, which is related to flexibility or stretchability. Some researchers proposed ultraviolet (UV) patterning as a strategy to optimize the recovery and relaxation of the PSAs used in flexible display applications [5]. The authors claimed that recovery and relaxation are important considerations for PSAs because the former is a prerequisite for stretchable/flexible devices as it prevents permanent (or plastic) deformation, while the latter significantly hinders the delamination that can lead to stress corrosion and cracking of the assembled display. Notably, recovery is defined as a restoring force of materials that depends on the change in entropy, which in turn relies on their molecular structure and length in accordance with the thermodynamics of rubber elasticity [14]. The term "relaxation" signifies that the stress applied in a system is relaxed through molecular motions, which in turn implies that the elongation of polymer chains or networks with low-crosslinking density (or percolation networks) vary with increasing entropy (i.e., less stress relaxation leads to higher elasticity) [15]. Thus, there is a tradeoff relationship between recovery and relaxation; therefore, achieving a highly optimized performance of PSAs with appropriate recovery and relaxation using conventional methods is a challenge.

Spurred by this challenge, we carried out fundamental research via a systematic investigation of the effect of chemical composition for the development of advanced and high-performance stretchable/flexible PSAs. Despite several studies being performed on the effect of the chemical composition of the PSAs on their adhesion [16-18], none of these reports related this effect to strain reversibility. Among the various acrylate monomers, 2-ethylhexyl acrylate (2EHA) and acrylic acid (AA) are generally used to offer flexibility or softness and adhesion to the PSAs, respectively $[12,19]$.
Table 1

Molar percentage of the comonomers used in the synthesis of acrylate-based PSAs.

\begin{tabular}{lll}
\hline Compositions & 2EHA (mol \%) & AA (mol \%) \\
\hline 1AA & 90 & 10 \\
3AA & 70 & 30 \\
5AA & 50 & 50 \\
7 AA & 30 & 70 \\
\hline
\end{tabular}

In this study, we first focused on the effect of chemical compositions on the physical performance of acrylate-based PSAs that were synthesized using different AA contents. Subsequently, the PSA exhibiting the best performance was used as the adhesive and subjected to further physical characterization to determine its stress relaxation, repetitive cyclability, light transmittance, and metal corrosiveness. Our findings are expected to offer design guidelines for advanced high-performance PSAs that can be utilized in a wide range of applications pertaining to display devices.

\section{Material and methods}

\subsection{Synthesis of acrylate-based PSAs}

Acrylate-based PSAs were synthesized by random copolymerization of acrylic monomers through a fast and facile UV curing process, namely: 2EHA (Sigma-Aldrich, USA) and AA (Sigma-Aldrich, USA). Varying ratios of these monomers were added to form a solution mixture (Table 1); $15 \mathrm{wt} \%$ of hydrophobic fumed silica as a filler (AEROSIL R 972, Evonik Industries, Germany) and $0.3 \mathrm{wt} \%$ (2 wt $\%$ of the fumed silica) of the dispersing agent (DISPERBYX-2158, BYK, Germany) were added to each mixture, and each chemical mixture was prepared by using a vortex mixer (Vortex-Genie 2, Scientific Industries, USA). Furthermore, $0.25 \mathrm{~mol} \%$ of 1,6-hexanediol diacrylate (HDDA, Alfa Aesar, USA) and $0.1 \mathrm{~mol} \%$ of phenylbis(2,4,6-trimethyl-benzoyl) phosphine oxide (TCI Chemicals, Japan)-with respect to the total monomer concentration-were used as a crosslinking agent and photoinitiator, respectively. The mixture was coated onto the release film (SG31, SKC, Republic of Korea) using a knife coating device (KP-3000VH, KIPAE, Republic of Korea). This was followed by the preparation of the laminate, which was then cured under a UV lamp (UVP B-100AP, AnalytikJena, Germany) for $30 \mathrm{~s}$ at a distance of $5 \mathrm{~cm}$. The thickness $(B)$ of the synthesized PSAs was $100 \pm 10 \mu \mathrm{m}$. However, thicker PSA specimens $(B=140 \pm 10 \mu \mathrm{m})$ were used for the stress relaxation and repetitive cycle tests for better handling.

\subsection{Peel adhesion test}

The as-prepared specimens with dimensions of $25 \times 300 \mathrm{~mm}^{2}$ $(\mathrm{W} \times \mathrm{L})$ were rolled twice across a SUS (stainless steel) panel using a $2.5 \mathrm{~kg}$ roller in the lengthwise direction. After $15 \mathrm{~min}$ of contact with the SUS at room temperature $\left(25^{\circ} \mathrm{C}\right)$, the peel adhesion test was performed using a universal testing machine (WL2100C, Withlab, Republic of Korea) at a crosshead speed of $300 \mathrm{~mm} \mathrm{~min}^{-1}$. The ASTM D3330 standard (Test Method A) was used to calculate the peel adhesion; the average of at least three repetitions was considered as the final result.

\subsection{Stress relaxation and repetitive cycle tests}

Stress relaxation and repetitive cycle tests were conducted using a universal testing machine (DAO-u1, DAO Technology, Republic of Korea) with dimensions of $13 \times 38 \mathrm{~mm}^{2}(W \times L)$ at room temperature. For testing the stress relaxation, the specimens were subjected to strains varying from $1 \%$ to $300 \%$ and relaxed for $1 \mathrm{~h}$. The stress relaxation ratio was calculated as the ratio between the decrease in stress after $1 \mathrm{~h}$ 
a

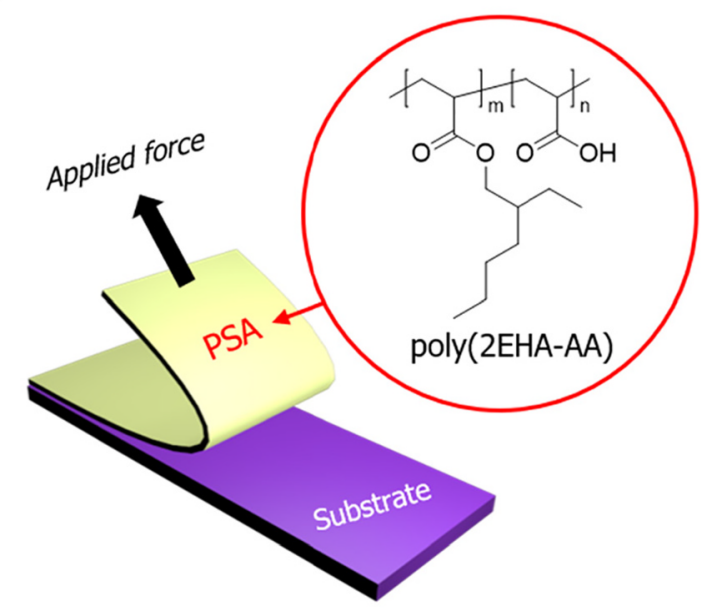

C

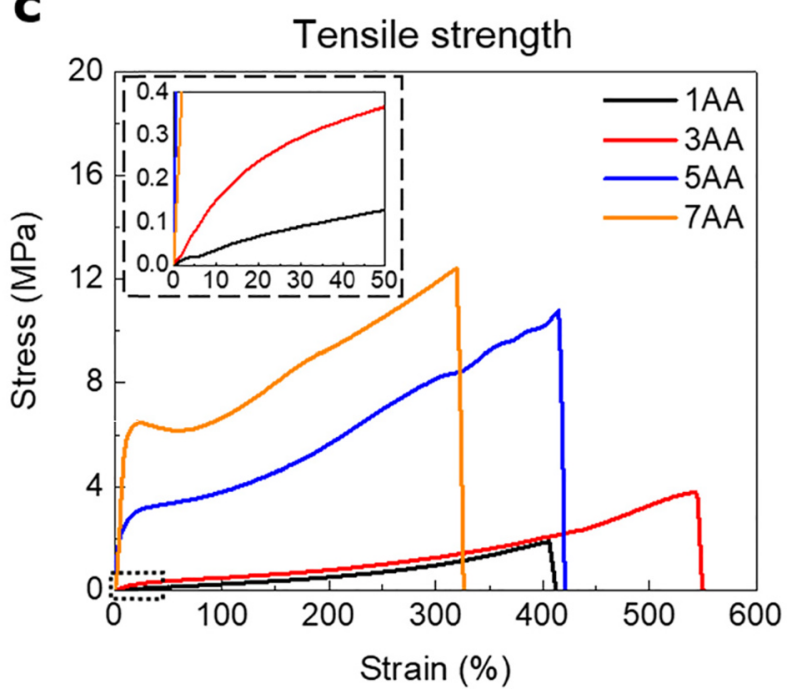

b
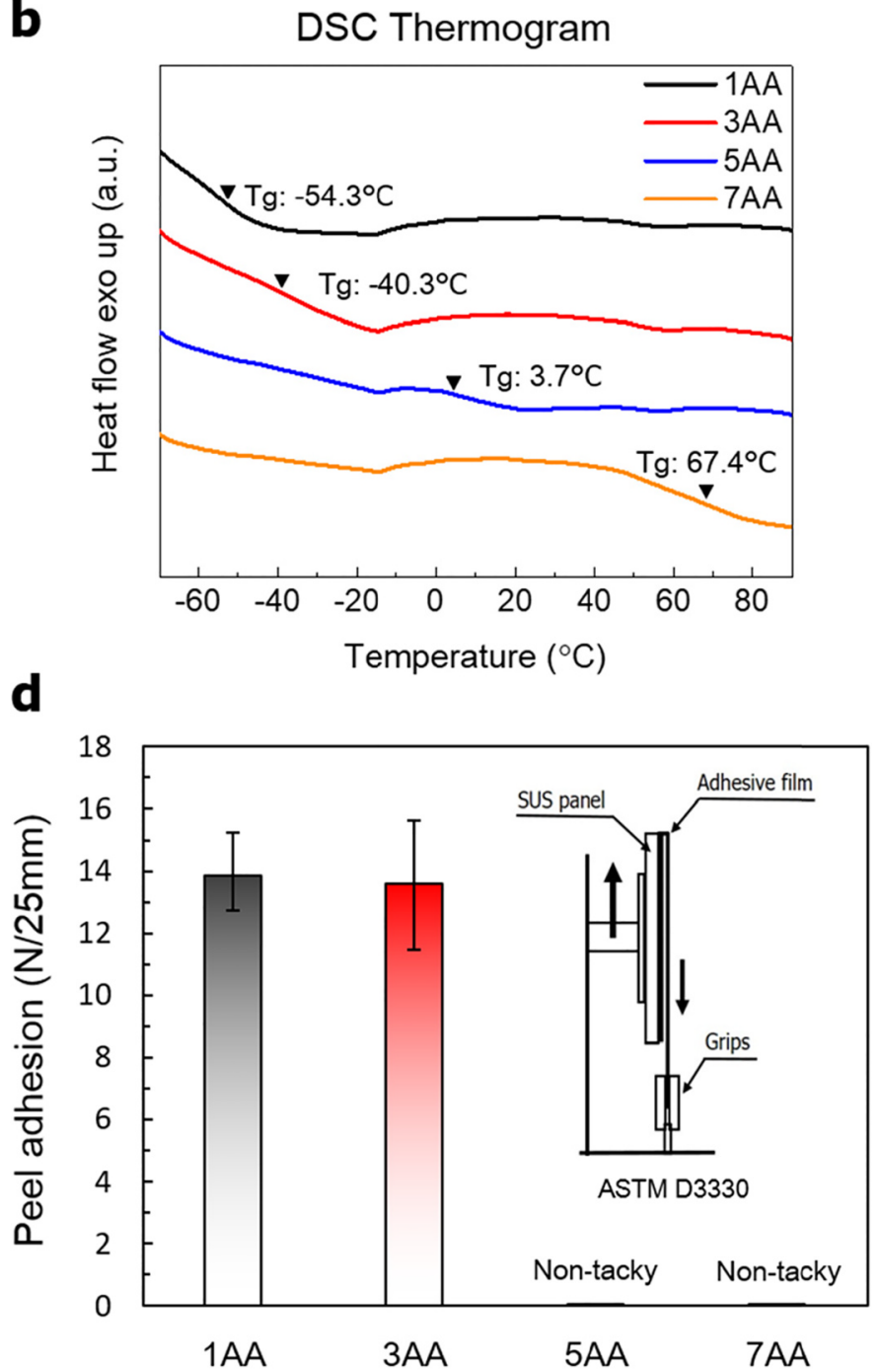

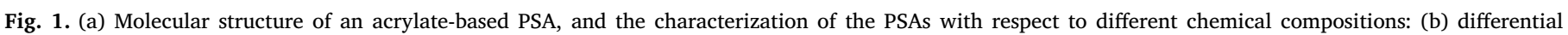
scanning calorimetry (DSC) thermogram, (c) typical stress-strain curves, and (d) peel adhesion.

of relaxation time and the initial stress at each applied strain. Plastic deformation was calculated by the percentage of permanent deformation after $1 \mathrm{~h}$ of relaxation, with respect to those of the pristine specimens. For the repetitive cycle test, the specimens were subjected to $25 \%$ strain at a crosshead speed of $500 \mathrm{~mm} \mathrm{~min}^{-1}$. Hysteresis loss was calculated by performing the numerical integration of the area between the loading and unloading curves [13].

\subsection{Transmittance measurement}

The transmittance values of the synthesized PSAs were measured by UV-Vis-NIR spectroscopy (Gary 5000, Varian, USA). The PSA-coated and bare glasses were used as the specimen and reference material, respectively. The scanned wavelength range to determine the transmittance was $350-700 \mathrm{~nm}$.

\subsection{Metal corrosiveness test}

The synthesized PSAs were applied on indium tin oxide (ITO)coated glass substrates (AMGtech, Republic of Korea) and the specimens were exposed to hygrothermal conditions $\left(50{ }^{\circ} \mathrm{C}\right.$ and $90 \%$ relative humidity) for four weeks. The surface resistance was measured using a four-point probe (CMT2000N, AIT, Republic of Korea) and the average surface resistance obtained from five different points of each specimen was reported.

\subsection{Gel fraction measurement}

The synthesized PSAs with dimensions of $13 \times 25 \mathrm{~mm}^{2}(W \times L)$ were weighed $\left(W_{\mathrm{i}}\right)$ and then immersed in tetrahydrofuran (THF) for $24 \mathrm{~h}$ at room temperature. The swollen PSAs were thoroughly dried for $24 \mathrm{~h}$ at room temperature, until they regained their original conformation. After drying, the PSAs were weighed $\left(W_{\mathrm{f}}\right)$ again. The gel fraction of the synthesized PSAs was calculated as follows: Gel fraction $(\%)=\left(W_{\mathrm{f}} / W_{\mathrm{i}}\right) \times 100$.

\section{Results and discussion}

\subsection{Characterization of the PSAs}

We first characterized the physical properties of the PSAs (Fig. 1(a)) prepared using different AA contents. The details of the thermal and mechanical analyses are available in the Supporting Information. We observed that the rigidity of the PSAs increases as the molar ratio of AA is increased from $10 \%$ to $70 \%$; this increase is represented by $1 \mathrm{AA}, 3 \mathrm{AA}$, 5AA, and 7AA, respectively. For example, the glass transition 


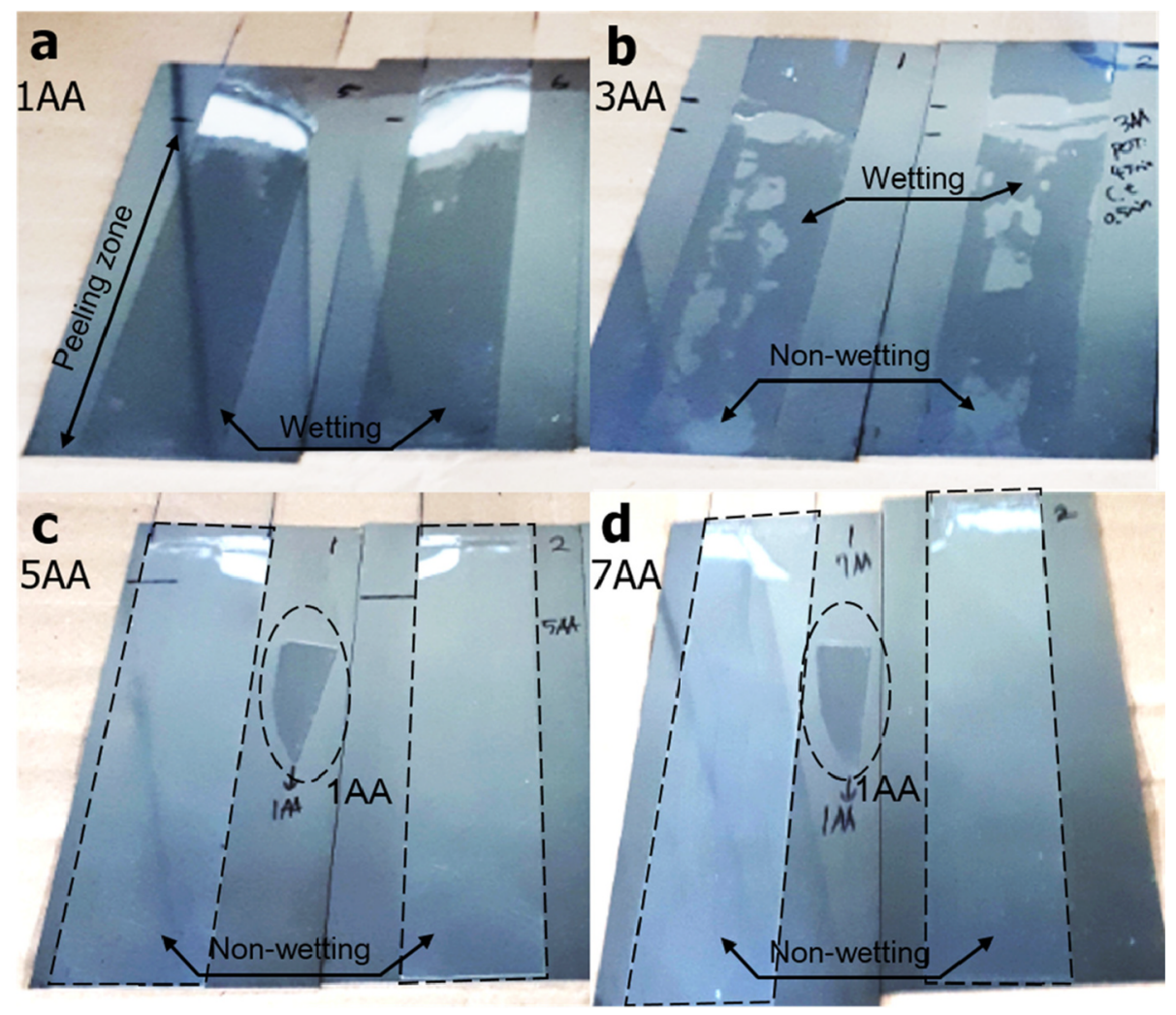

Fig. 2. Wetting images of the acrylic acid (AA)-incorporated PSAs with varying chemical compositions: (a) 1AA, (b) 3AA, (c) 5AA, and (d) 7AA; 1AA was included in (c) and (d) to compare the wetting behavior.

temperatures of the PSAs increase with the increasing AA content (Fig. 1(b)), thereby indicating fewer molecular motions such as bond rotation at room temperature; this result is in accordance with the free volume theory. This hindrance of molecular movement causes rigidity and is congruous with the mechanical behavior of the AA-incorporated specimens, which become stiffer as the concentration of AA increases (Fig. 1(c)). Fig. 1(d) shows the peel adhesion of the PSAs at different chemical compositions; 5AA and 7AA exhibit almost zero peel adhesion, whereas 1AA and 3AA exhibit a high peel adhesion at $\sim 13.8 \mathrm{~N} /$ $25 \mathrm{~mm}$, which is approximately $65 \%$ higher than that of the widely used 'Scotch tape' $(8.34 \mathrm{~N} / 25 \mathrm{~mm}$ ) (PK65 $48 \times 40$ transparent, $3 \mathrm{M}$, USA) [13].

Furthermore, the wetting images and rheological properties of the AA-incorporated PSAs were investigated to explain the relation between the AA content and peel adhesion. All adhesives must achieve intimate wetting with their substrates to maximize their adhesion performance. As the PSAs behave more like soft solids rather than liquids during the wetting process, their wettability is governed by their moduli; here, a lower modulus was observed to promote wetting [20]. Although the adhesive is wetted and forms interfacial bonds with the substrate, it must be able to resist fracture and debonding to effectively perform its function. Hence, fracture toughness is an important property for an adhesive, which depends on its ability to dissipate energy via a yielding deformation near the crack tip [20]. Generally, the loss modulus $\left(G^{\prime \prime}\right)$ of an adhesive indicates the amount of energy absorbed through deformation during peeling [21]. Fig. 2 suggests that increasing the AA concentration is detrimental to the wettability of the adhesives in contact with SUS. The aforementioned explanation is also supported by the behavior of the storage (Fig. S1(a)) and Young's moduli (Table S1) of the prepared PSAs, and sheds light on the following criterion for intimate wetting of an adhesive in this system: the storage modulus of the PSA must be less than $55 \mathrm{kPa}$ at $1 \mathrm{~Hz}$. This criterion agrees well with all PSAs that have been previously demonstrated to exhibit the best adhesion [22].

In terms of the energy dissipation characterized by the loss moduli (Fig. S1(b)) of the PSAs, 3AA is expected to exhibit higher peel adhesion than that of 1AA. Although 3AA can dissipate energy more vigorously than 1AA, the "non-wetting" spots of 3AA (Fig. 2(b)) result in a decrease in its total contact area, thereby leading to the failure of the bulk energy dissipation mechanism. Furthermore, a recent study had demonstrated that AA-incorporated PSAs establish strong interfacial bonds, which are induced by the contact with the metallic adherend through molecular reorientation and polymer chain rearrangement [23]. Thus, a greater number of acid-base interactions with the per unit area of "wetting" spots in the substrate can be achieved in 3AA as it has more acid moieties $(30 \mathrm{~mol} \%)$ than those of $1 \mathrm{AA}(10 \mathrm{~mol} \%)$; this is possibly the reason for the comparable peel adhesion results observed for 1AA and 3AA. This overview leads to the primary conclusion that an intimate wetting between the PSA and its adherend is a prerequisite for high adhesion performance, unless the adhesive behaves like a liquid, which can be modulated through the incorporated AA content. As it exhibited the best adhesion performance among the compositions in terms of wetting and peel adhesion, 1AA was further investigated in order to determine its viscoelastic properties and evaluate its eligibility for a wide range of electronic applications.

\subsection{Stress relaxation and repetitive cycle tests}

Owing to the fact that strain is unchangeable after stretching, no macroscopic movement of the specimen is possible during the stress relaxation test; therefore, stress relaxation can only occur at the molecular level. There are several mechanisms to explain the relaxation process, such as viscous flow, molecular relaxation, and disentanglement or bond interchange (e.g., vitrimers) of dynamic crosslinks $[24,25]$. These processes are such that they attain equilibrium under an applied stress [26]. Since an irreversible percolation network is 


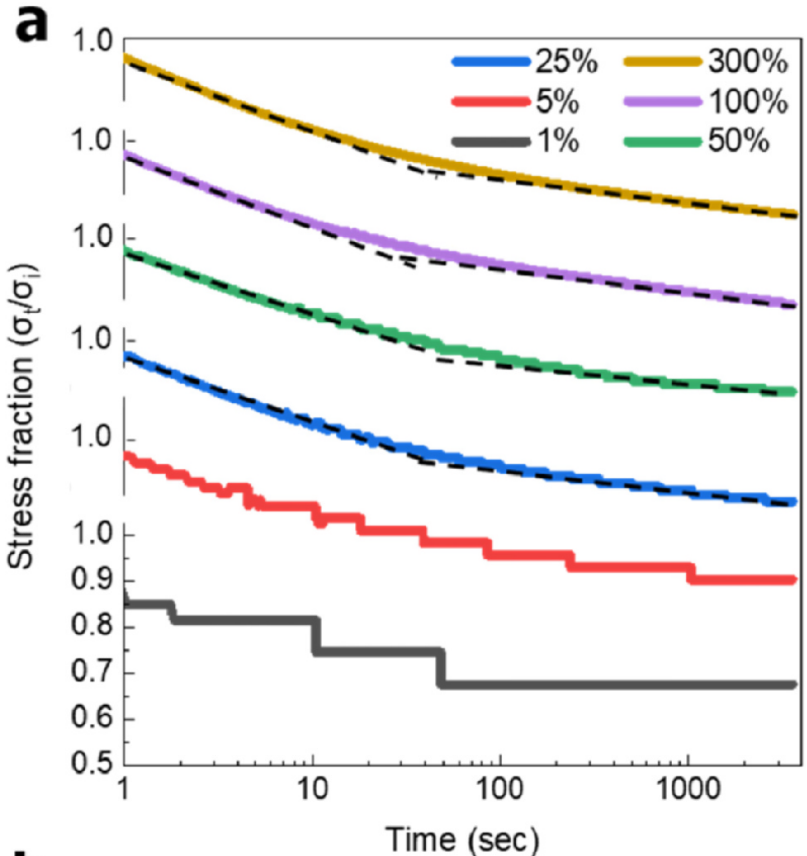

b

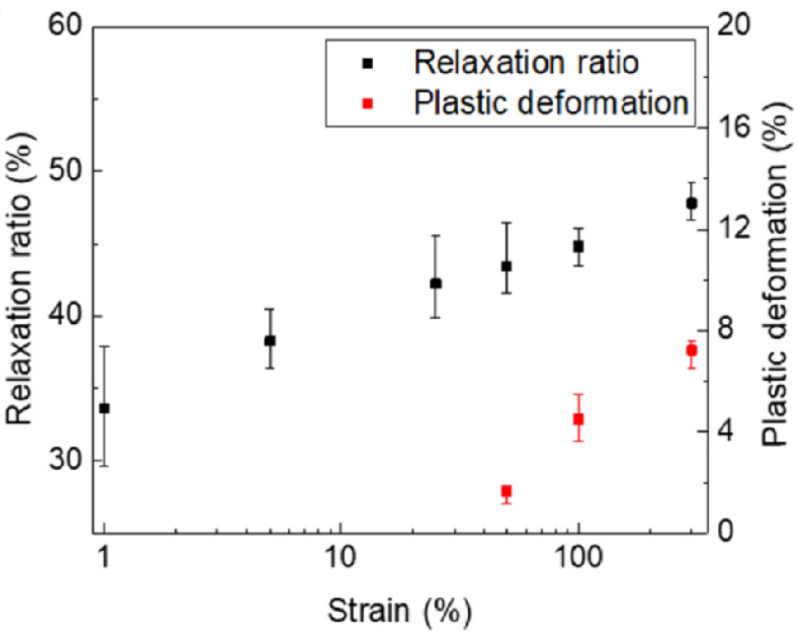

Fig. 3. Stress relaxation of $1 \mathrm{AA}$ under various strains for $1 \mathrm{~h}$ : (a) semilogarithmic plot of $1 \mathrm{AA}$ under different strains, where $\sigma_{\mathrm{i}}$ represents the maximum stress and $\sigma_{t}$ indicates the stress at time $t$; (b) calculated relaxation ratio and plastic deformation of $1 \mathrm{AA}$ with respect to strain. The stair-like stress-time curves of $1 \mathrm{AA}$ at $1 \%$ and $5 \%$ are attributed to the resolution of the $10 \mathrm{kgf}$ load cell in addition to the low stiffness of $1 \mathrm{AA}$, which limits the linear fitting.

established in 1AA by using HDDA, the explicit viscous flow caused by a linear polymer-chain slip or dynamic covalent bond interchange is not favorable. Fig. 3(a) shows the stress relaxation test results for 1AA with various strains as a function of time. A measure of the stress fraction was achieved by dividing the stress at time $t\left(\sigma_{t}\right)$ by the maximum stress $\left(\sigma_{\mathrm{i}}\right)$ (the reference stress). The time zero $(0 \mathrm{~s})$ was set as the time at which the stress values of the specimens were maximum. We observe that the experimental results consist of two relaxation time constants, which appear as two straight lines in the semilogarithmic plots. A greater slope is achieved for a time period of less than about $1 \mathrm{~min}$ and a smaller slope is attained for a greater time period; this result corresponds to the stress value against the logarithmic time scale of the carbon black-reinforced natural rubber composites [27]. The slopes of the two linear lines are available in the Supporting Information (Table S2). The regimes distinguished by two linear sections imply two distinct relaxation mechanisms of the fumed silica reinforced adhesives. The stress relaxation of a crosslinked polymer network was proposed to occur via a retracting mechanism (a concept of reptation), wherein the unassociated chain ends (or the dangling chain) diffuse toward the chain ends that are attached to the network $[28,29]$. The deformation of the filler phase structure was suggested to describe the early relaxation process of the filler-reinforced rubber vulcanizates [27]. Based on these proposed relaxation mechanisms, we postulated that the viscoelastic relaxation of $1 \mathrm{AA}$ can be attributed to the deformation of the filler phase structure built from the fumed silica particles, which is dominant within the first $1 \mathrm{~min}$ of the relaxation; this is followed by the retraction of the free chain ends toward chain ends that are attached to the network.

The stress relaxation ratio and plastic deformation of $1 \mathrm{AA}$ at various strains are shown in Fig. 3(b), and their conformation after the test is shown in Fig. 4. The results indicate that the relaxation ratio and plastic deformation are proportional to the strain. The stress-time plots of 1AA at various strains are represented in Fig. S2. As the elasticity of rubber is generally known to be driven by entropy change, we observe that all specimens subjected to strains less than or equal to $25 \%$ exhibit a complete recovery, even though the recovery time depends on the strain (Fig. 4(a) and (b)). The recovery indicates that 1AA displays an elastic regime of up to $25 \%$ strain. On the other hand, a partial recovery is observed for the specimens with strains higher than $25 \%$, which stops further recovery after $20 \mathrm{~min}$ ( $t=20 \mathrm{~min}$ ) of the stress relaxation test (Fig. 4(c) and (d)). Furthermore, Fig. 4(a) and (b) indicate the conserved degree of disorder to a certain extent despite elongation, which enables complete recovery according to the thermodynamics of rubber elasticity. The temporal difference of Fig. 4(a) and (b) suggest that the acid moieties in 1AA are more likely to cause the reversible hydrogen bond formation under higher strain, resulting in a stronger resistance to retraction. This speculation can be driven by a shorter inter-chain distance based on the description of the Poisson ratio of the polymeric materials, which represents a contraction that is perpendicular to the longitudinal elongation [30]. The plastic deformation observed at strains higher than $25 \%$ can be due to the balance between the entropic restoring force and reversible hydrogen bonds, which prevents retraction.

Three conditions must be fulfilled to achieve rapid strain reversibility, namely: (1) the polymeric materials should be amorphous so that they can thermodynamically favor strain reversibility; (2) the number of intermolecular forces hindering the polymer chain retraction (e.g., hydrogen bonds) should be minimum; and (3) crosslinking is required to provide percolation network, which prevents relative translational motion [31]. All these criteria can lead to elastomer-like performance, which minimizes energy loss in the form of heat during loading and unloading. The stress-strain responses of 1AA against the dynamic or repetitive mechanical stimulus are shown in Fig. 5. The choice of the $25 \%$ strain was based on the above stress relaxation results that show a complete recovery of the specimen. We observe that the time required for a complete recovery (Fig. 5(e)) after a 100-cycle test is approximately $2 \mathrm{~min}$, which is around 10 times faster than that obtained from the stress relaxation test at the same strain. This can be ascribed to the instant dwell time at the maximum strain, which limits the formation of reversible hydrogen bonds among the polymer chains.

Ten sets of sequential and repetitive 100-cycle tests that were performed at 2 min intervals confirmed that $1 \mathrm{AA}$ can maintain its conformational recovery as well as mechanical properties (Fig. 5(e)). Nevertheless, the recovery time must be minimized for user convenience; for instance, if the displays of contemporary devices such as flexible smart watches and foldable mobile phones (e.g., SAMSUNG Galaxy Fold, SAMSUNG Galaxy Z Flip, and HUAWEI Mate X) were not able to achieve rapid strain reversibility, they could be disfigured by wrinkles or a wavy surface, causing discomfort to the users. Hence, we implemented a prestrain strategy to accomplish instantaneous strain reversibility at $15 \%$ strain (a video of an instantaneously reversible 1AA at $10 \%$ prestrain is available in Supporting Information). Fig. 5(f) displays the stress-strain curves of $1 \mathrm{AA}$ at $10 \%$ prestrain for a 100 -cycle 
a

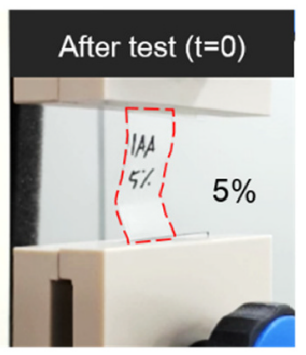

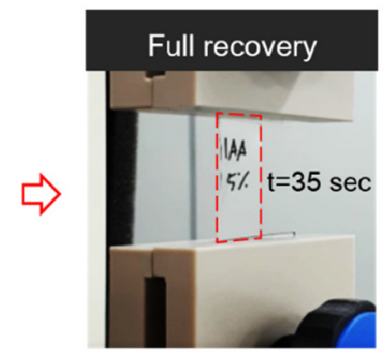

b
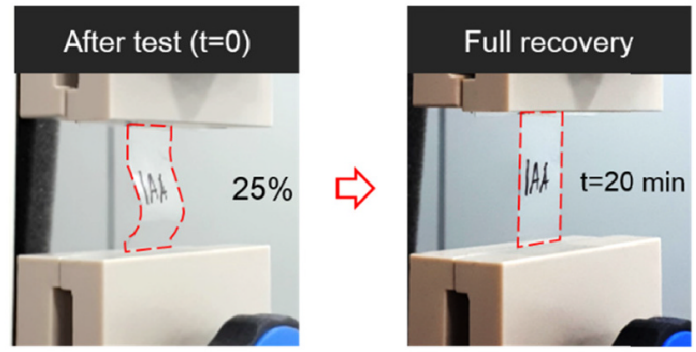

C
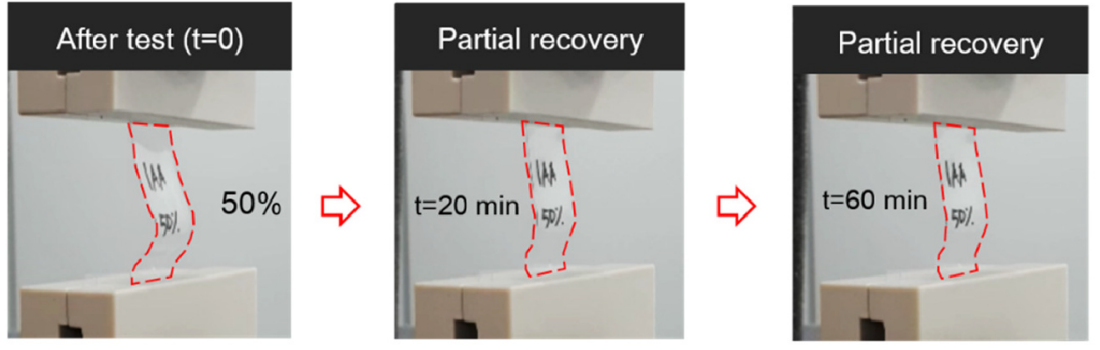

d
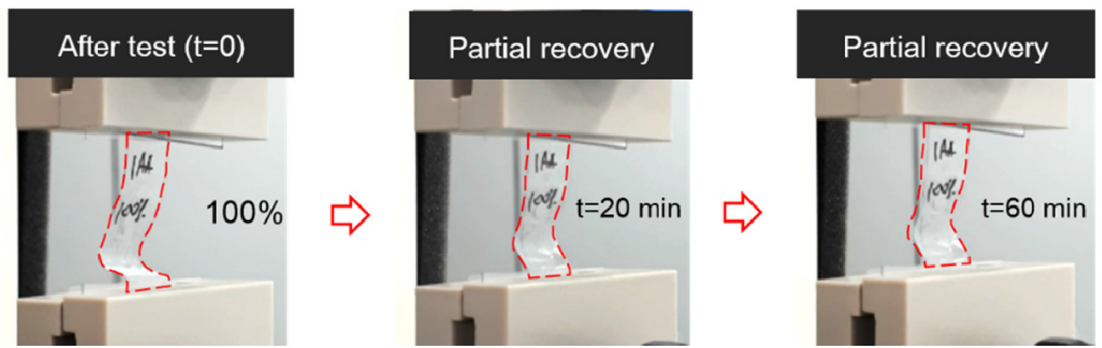

Fig. 4. Specimens observed immediately after the stress relaxation test $(t=0)$ subjected to: (a) $5 \%$, (b) $25 \%$, (c) $50 \%$, and (d) $100 \%$ strain. The $300 \%$ strained specimens exhibited a worse recovery than those subjected to $100 \%$ strain.
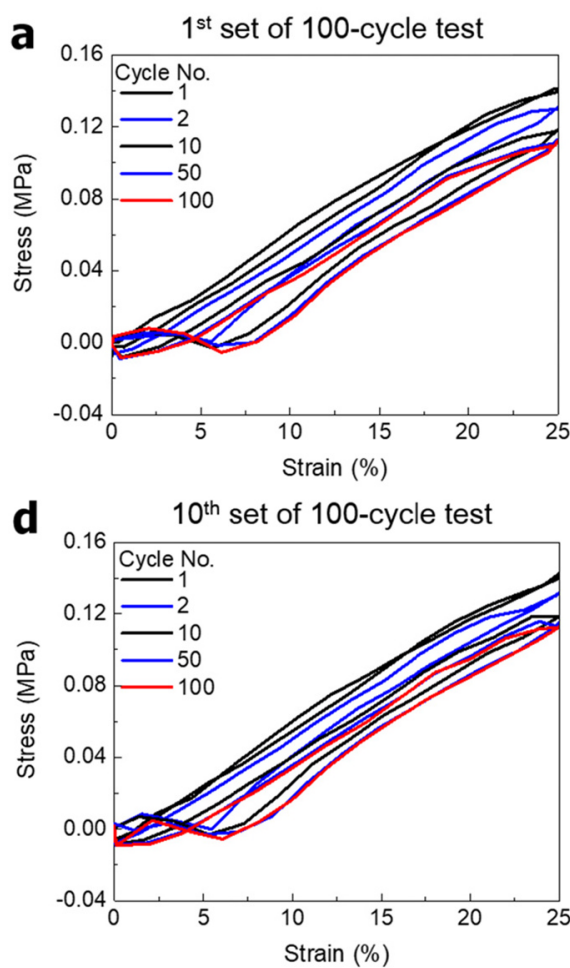

b $3^{\text {rd }}$ set of 100-cycle test

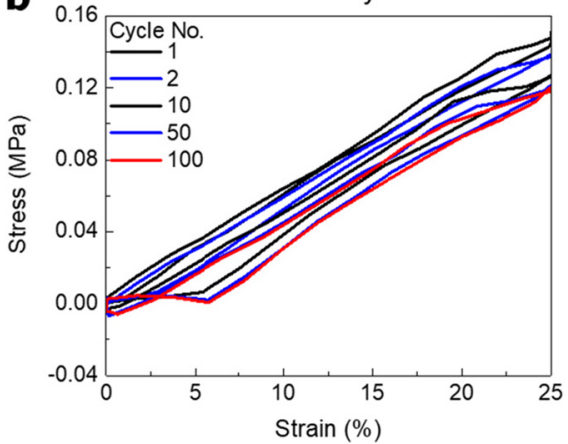

e

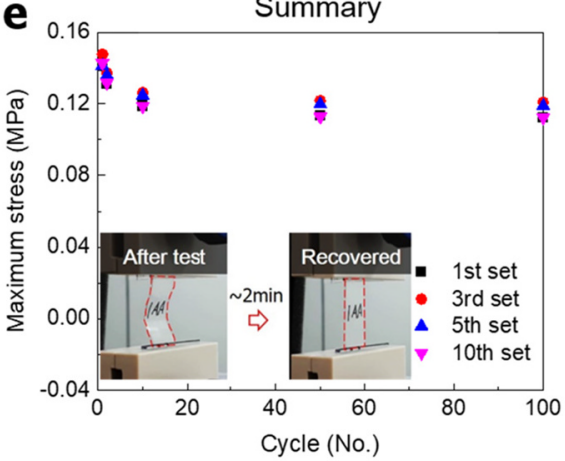

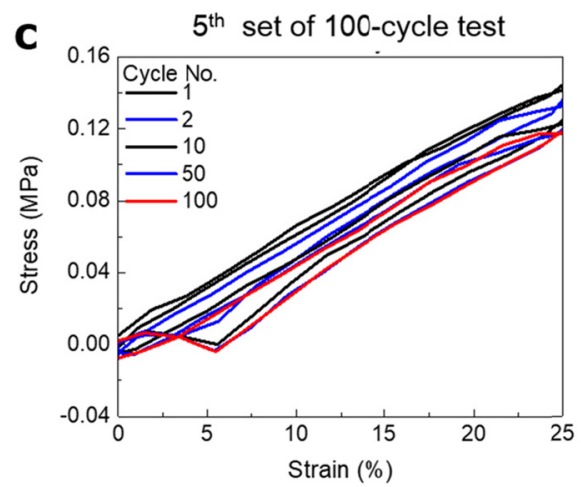

f

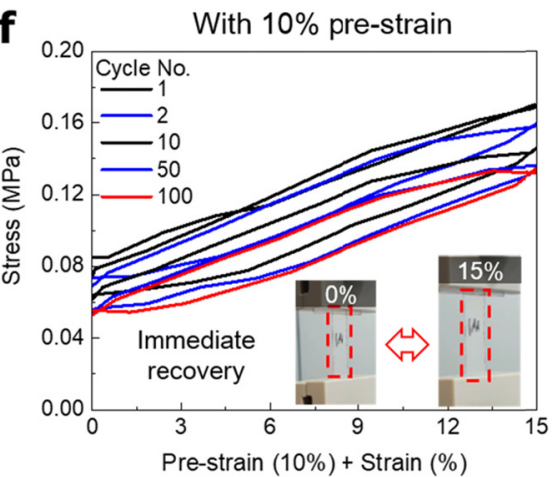

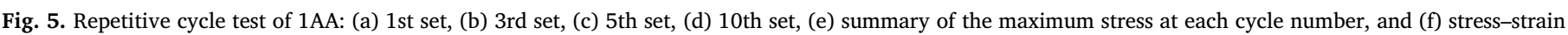

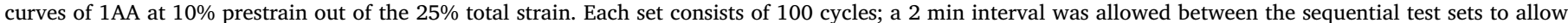
complete recovery. 


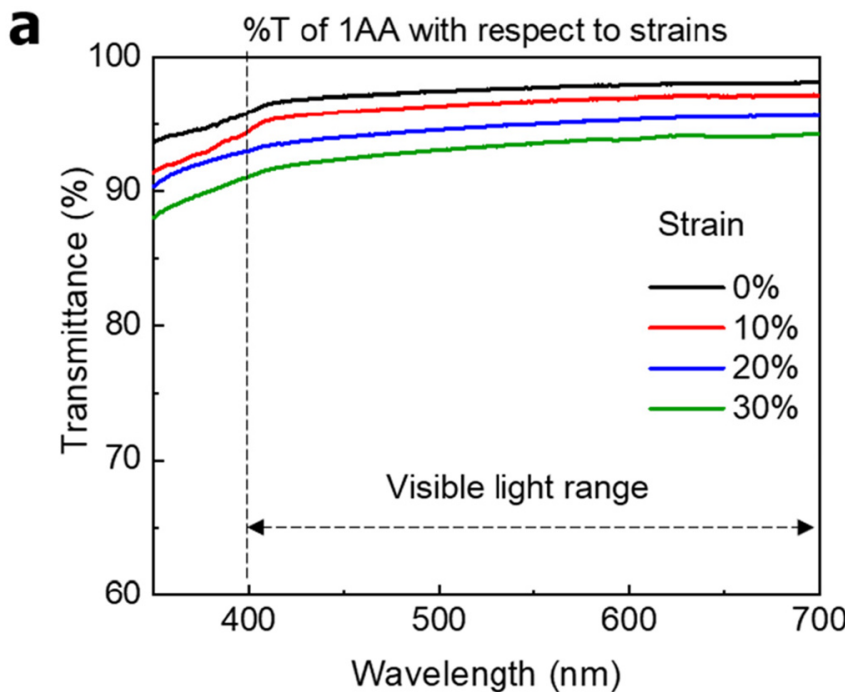

C

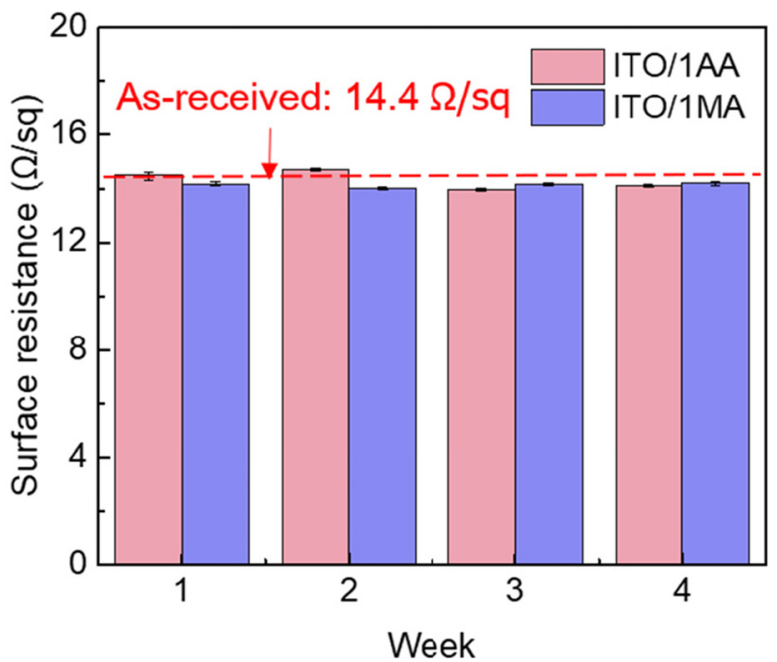

b

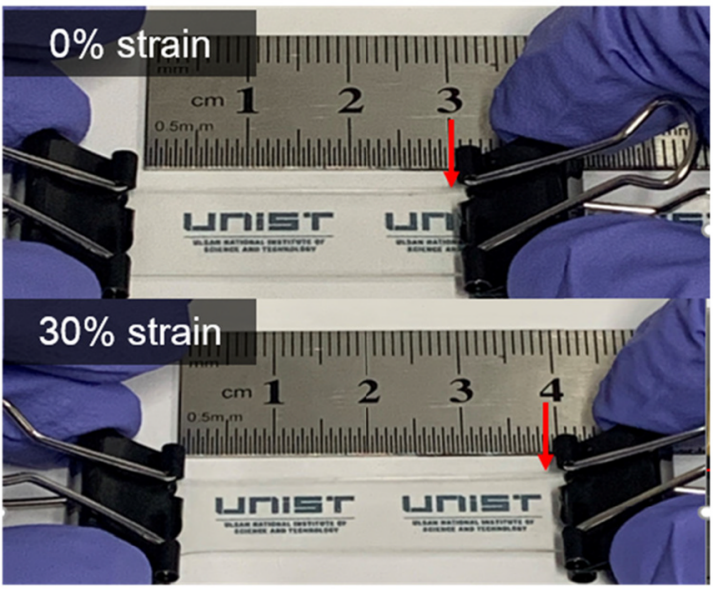

d
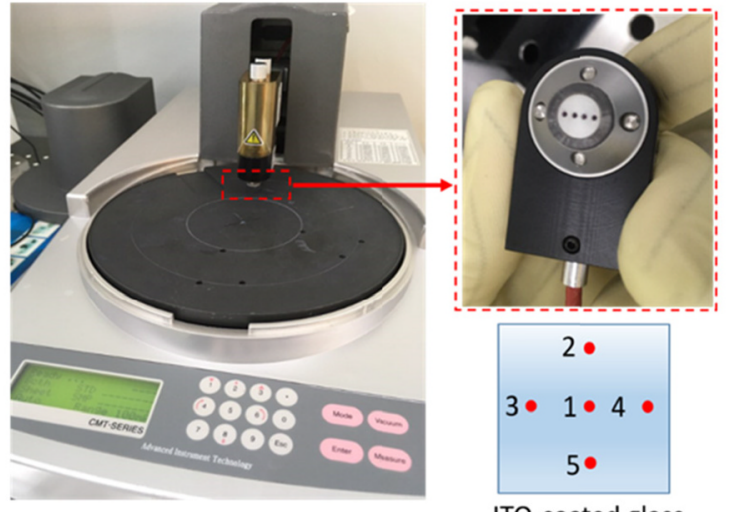

ITO-coated glass

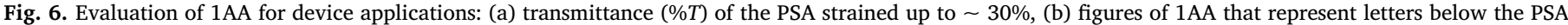

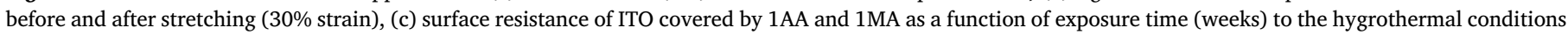
of $50{ }^{\circ} \mathrm{C}$ and $90 \%$ relative humidity, and (d) surface resistance measurement using the four-point probe.

test. A practically identical hysteresis loss over the cycles $\left(0.219 \pm 0.014 \mathrm{~J} / \mathrm{cm}^{3}\right.$, except in the 1 st cycle) reflects its elastomerlike behavior [13].

\subsection{Evaluation for device applications}

The transmittance measurement of the PSAs at varying AA contents is shown in Fig. S3. An evaluation of 1AA for practical device applications was conducted in terms of light transmittance and compatibility to a corrosion-sensitive layer. Fig. 6(a) and (b) show the transmittance and visual transparency of 1AA, respectively; it exhibits high transmittance $(>90 \% T)$ over the visible light range under strains of up to $30 \%$, indicating that $1 \mathrm{AA}$ is highly transparent during repetitive cyclic motion with rapid retraction driven by prestrain. The decline in $\% T$ with increasing strain can be attributed to the change in the polymer structure (from a random coil to linear chain), which possibly increases the crystallinity or the fluctuation of the filler phase structure due to the reorientation or alignment of the filler $[32,33]$.

Although the AA-incorporated PSAs are well known for their excellent adhesion performance, some reports have described that the addition of carboxylic acid to soft adhesives can lead to the corrosion of metal substrates $[8,34]$. Therefore, we evaluated the compatibility of $1 \mathrm{AA}$ with a corrosion-sensitive layer (ITO) by applying 1AA and 1MA (composed of $10 \mathrm{~mol} \%$ of methyl acrylate) on ITO-coated glass substrates, and subjected to the specified hygrothermal conditions for four weeks; 1MA was used as a reference for comparing the acid effects. Fig. 6(c) shows the surface resistance of the ITO substrates covered by the PSAs; we observe that their surface resistance is invariant as compared to the "As-received" substrates, regardless of the functional groups (carboxylic acid or methyl group) being a function of the exposure time to the hygrothermal conditions of $50{ }^{\circ} \mathrm{C}$ and $90 \%$ relative humidity. These results indicate a promising compatibility between $1 \mathrm{AA}$ and the corrosion-sensitive layer; moreover, these results are comparable to the outcome of the US patent on the electrical resistance consistency of ITO after aging [35]. The underlying mechanism for ITO corrosion is contamination due to impurities [36]. The metal corrosion resistance of 1AA can be related to its high gel fraction $(99.2 \pm 0.5 \%)$, which hardly leaves any residual acrylic monomer on the ITO substrates. Thus, this evaluation demonstrates the eligibility of 1AA for stretchable/flexible electronic devices with advanced properties.

\section{Conclusions}

The physical properties of PSAs with varying AA contents were successfully characterized in this study. The results indicate that higher AA concentrations can result in stiffer and more translucent PSAs, 
which not only lead to poor adhesion performance but also to unfavorable wetting of the substrate. To evaluate the adhesion performance for electronic device applications, the PSA with $10 \mathrm{~mol} \%$ AA was subjected to a viscoelastic test. The results showed that under a static stimulus, the relaxation ratio was strain-dependent, thereby suggesting elastic behavior up to a certain elongation. Furthermore, its viscous component becomes dominant under higher strains, which was quantitatively estimated using plastic deformation calculations. The conformational and mechanical recoveries of the designated PSA emphasize its advantage for stretchable adhesive design. Moreover, our prestrained PSAs enable instantaneous strain reversibility under $25 \%$ strain, indicating the promising materials for stretchable/flexible applications. The evaluation substantiates high optical clarity and excellent compatibility of the AA-incorporated PSAs with a corrosionsensitive layer. Thus, our results provide design guidelines to achieve both relaxation and recovery with high adhesion performance and metal corrosion resistance, which make a positive contribution to the development of advanced adhesives for applications related to display devices.

\section{Declaration of Competing Interest}

The authors declare that they have no known competing financial interests or personal relationships that could have appeared to influence the work reported in this paper.

\section{Acknowledgements}

This work was supported by the Industrial Strategic Technology Development Program (No. 20011089, 'Development of thermal conductive film and bonding material for flexible display with $300 \mathrm{WmK}$ in plane thermal conductivity') funded by the Ministry of Trade, Industry \& Energy (MOTIE, Korea); the Basic Science Research Program (NRF2019R1A2C2005854) funded by the National Research Foundation (NRF) of Korea; and the Basic Science Institute Research Fund (1.200049.01) of Ulsan National Institute of Science and Technology (UNIST).

\section{Appendix A. Supplementary data}

Supplementary data to this article can be found online at https:// doi.org/10.1016/j.cej.2020.126800.

\section{References}

[1] J.H. Koo, D.C. Kim, H.J. Shim, T.-H. Kim, D.-H. Kim, Flexible and stretchable smart display: materials, fabrication, device design, and system integration, Adv. Funct. Mater. 28 (2018) 1801834.

[2] S. Lim, D. Son, J. Kim, Y.B. Lee, J.-K. Song, S. Choi, D.J. Lee, J.H. Kim, M. Lee, T. Hyeon, D.-H. Kim, Transparent and stretchable interactive human machine interface based on patterned graphene heterostructures, Adv. Funct. Mater. 25 (2015) 375-383.

[3] D.-H. Kim, N. Lu, R. Ma, Y.-S. Kim, R.-H. Kim, S. Wang, J. Wu, S.M. Won, H. Tao, A. Islam, K.J. Yu, T.-I. Kim, R. Chowdhury, M. Ying, L. Xu, M. Li, H.-J. Chung, H. Keum, M. McCormick, P. Liu, Y.-W. Zhang, F.G. Omenetto, Y. Huang, T. Coleman, J.A. Rogers, Epidermal electronics, Science 333 (2011) 838.

[4] S.R. Krishnan, C.-J. Su, Z. Xie, M. Patel, S.R. Madhvapathy, Y. Xu, J. Freudman, B. Ng, S.Y. Heo, H. Wang, T.R. Ray, J. Leshock, I. Stankiewicz, X. Feng, Y. Huang, P. Gutruf, J.A. Rogers, Wireless, battery-free epidermal electronics for continuous, quantitative, multimodal thermal characterization of skin, Small 14 (2018) 1803192.

[5] J.-H. Back, D. Baek, K.-B. Sim, G.-Y. Oh, S.-W. Jang, H.-J. Kim, Y. Kim, Optimization of recovery and relaxation of acrylic pressure-sensitive adhesives by using UV patterning for flexible displays, Ind. Eng. Chem. Res. 58 (2019) 4331-4340.
[6] J.-G. Lee, G.-S. Shim, J.-W. Park, H.-J. Kim, K.-Y. Han, Kinetic and mechanical properties of dual curable adhesives for display bonding process, Int. J. Adhes. Adhes. 70 (2016) 249-259.

[7] J.H. Lee, T.H. Lee, K.S. Shim, J.W. Park, H.J. Kim, Y. Kim, S. Jung, Effect of crosslinking density on adhesion performance and flexibility properties of acrylic pressure sensitive adhesives for flexible display applications, Int. J. Adhes. Adhes. 74 (2017) 137-143.

[8] C.-H. Park, S.-J. Lee, T.-H. Lee, H.-J. Kim, Characterization of an acrylic polymer under hygrothermal aging as an optically clear adhesive for touch screen panels, Int. J. Adhes. Adhes. 63 (2015) 137-144.

[9] I. Benedek, M.M. Feldstein, Handbook of pressure-sensitive adhesives and products:-three volume set, CRC Press, 2008.

[10] C. Creton, Pressure-sensitive adhesives: an introductory course, Mater. Res. Soc. Bull. 28 (2003) 434-439.

[11] N. Ishikawa, M. Furutani, K. Arimitsu, Pressure-sensitive adhesive utilizing molecular interactions between thymine and adenine, J. Polym. Sci., Part A: Polym. Chem. 54 (2016) 1332-1338.

[12] Z. Czech, Synthesis and cross-linking of acrylic PSA systems, J. Adhes. Sci. Technol. 21 (2007) 625-635.

[13] J.H. Lee, M.H. Myung, M.J. Baek, H.-S. Kim, D.W. Lee, Effects of monomer functionality on physical properties of 2-ethylhexyl acrylate based stretchable pressure sensitive adhesives, Polym. Test. 76 (2019) 305-311.

[14] D.I. Bower, Mechanical properties I - time-independent elasticity, An Introduction to Polymer Physics, Cambridge University Press, Cambridge, 2002, pp. 162-186.

[15] N.S. Salem, D.C. Watts, E.C. Combe, Stress relaxation of elastomers, Dent. Mater. 3 (1987) 37-39.

[16] A. Falsafi, M. Tirrell, A.V. Pocius, Compositional effects on the adhesion of acrylic pressure sensitive adhesives, Langmuir 16 (2000) 1816-1824.

[17] M.D. Gower, R.A. Shanks, Acrylic acid level and adhesive performance and peel master-curves of acrylic pressure-sensitive adhesives, J. Polym. Sci., Part B: Polym. Phys. 44 (2006) 1237-1252.

[18] D. Ghim, J.H. Kim, Effects of composition and layer thickness of a butyl acrylate/ acrylic acid copolymer on the adhesion properties, Korean J. Chem. Eng. 33 (2016) 707-710.

[19] Y. Peykova, O.V. Lebedeva, A. Diethert, P. Müller-Buschbaum, N. Willenbacher, Adhesive properties of acrylate copolymers: effect of the nature of the substrate and copolymer functionality, Int. J. Adhes. Adhes. 34 (2012) 107-116.

[20] D. Yarusso, Adhesion Science and Engineering-The Mechanics of Adhesion, edited by DA Dillard and AV Pocius, Elsevier, Amsterdam, 2002.

[21] F.A. Mazzeo, Characterization of pressure sensitive adhesives by rheology, TA Instruments report RH 082 (2002) 1-8.

[22] M.M. Feldstein, R.A. Siegel, Molecular and nanoscale factors governing pressuresensitive adhesion strength of viscoelastic polymers, J. Polym. Sci., Part B: Polym. Phys. 50 (2012) 739-772.

[23] J.H. Lee, D.W. Lee, Contact-induced molecular rearrangement of acrylic acid-incorporated pressure sensitive adhesives, Appl. Surf. Sci. 500 (2020) 144246.

[24] Polymer Properties Database, Stress relaxation processes, Access date: July 18, 2019, doi: http://polymerdatabase.com/polymer\%20physics/Relaxation.html.

[25] M. Chen, L. Zhou, Y. Wu, X. Zhao, Y. Zhang, Rapid stress relaxation and moderate temperature of malleability enabled by the synergy of disulfide metathesis and carboxylate transesterification in epoxy vitrimers, ACS Macro Lett. 8 (2019) 255-260.

[26] D.I. Bower, Morphology and motion, An Introduction to Polymer Physics, Cambridge University Press, Cambridge, 2002, pp. 117-161.

[27] C.I. MacKenzie, J. Scanlan, Stress relaxation in carbon-black-filled rubber vulcanizates at moderate strains, Polymer 25 (1984) 559-568.

[28] S. Mitra, S. Chattopadhyay, A.K. Bhowmick, Dynamic stress relaxation behavior of nanogel filled elastomers, J. Polym. Res. 18 (2011) 489-497.

[29] M. Doi, S.F. Edwards, The Theory of Polymer Dynamics, Oxford University Press, Oxford, 1986.

[30] J.A. Rinde, Poisson's ratio for rigid plastic foams, J. Appl. Polym. Sci. 14 (1970) 1913-1926.

[31] R.A. Shanks, I. Kong, General purpose elastomers: structure, chemistry, physics and performance, Advances in Elastomers I, Springer, 2013, pp. 11-45.

[32] W.J. Song, J. Park, D.H. Kim, S. Bae, M.J. Kwak, M. Shin, S. Kim, S. Choi, J.H. Jang, T.J. Shin, Jabuticaba-inspired hybrid carbon filler/polymer electrode for use in highly stretchable aqueous Li-ion batteries, Adv. Energy Mater. 8 (2018) 1702478.

[33] K. Yamaguchi, J.J.C. Busfield, A.G. Thomas, Electrical and mechanical behavior of filled elastomers. I. The effect of strain, J. Polym. Sci., Part B: Polym. Phy. 41 (2003) 2079-2089.

[34] K.A. Sierros, N.J. Morris, K. Ramji, D.R. Cairns, Stress-corrosion cracking of indium tin oxide coated polyethylene terephthalate for flexible optoelectronic devices, Thin Solid Films 517 (2009) 2590-2595.

[35] A.I. Everaerts, J. Xia, Adhesives compatible with corrosion sensitive layers, United States Patents, 3M Innovative Properties Company, US 20100040842A1, 2010.

[36] W.S. Leung, Y.C. Chan, S.M. Lui, A study of degradation of indium tin oxide thin films on glass for display applications, Microelectron. Eng. 101 (2013) 1-7. 